\title{
The Main Injector Particle Physics Experiment (MIPP FNAL E-907) at Fermilab - status and plans
}

\author{
Rajendran Raja ${ }^{a}$ \\ ${ }^{\text {a }}$ Fermilab, P.O. Box 500, Batavia, ILlinois 60510, U.S.A
}

We describe the status of the Main Injector particle production Experiment (MIPP) at Fermilab which has to date acquired 18 million events of particle interactions using $(5 \mathrm{GeV} / \mathrm{c}-120 \mathrm{GeV} / \mathrm{c}) \pi^{ \pm}, K^{ \pm}$and $p^{ \pm}$beams on various targets. We describe plans to upgrade the data acquisition speed of MIPP to make it run 100 times faster which will enable us to obtain particle production data of unprecdented quality and statistics on a wide variety of nuclear targets including nitrogen which is of importance to cosmic ray physics.

\section{Current Status of the MIPP Experi- ment}

We describe status report on the MIPP [1] experiment and its performance to date. MIPP is situated in the Meson Center beamline at Fermilab. It received approval 2] in November 2001 and has installed and operated both the experiment and a newly designed secondary beamline in the interim. It received its first beams in March 2004, had an engineering run to commission the detector in 2004 and had its physics data-taking run in the period January 2005-March 2006. The experiment is currently busy analyzing its data.

MIPP is designed primarily as an experiment to measure and study in detail the dynamics associated with non-perturbative strong interactions. It has nearly $100 \%$ acceptance for charged particles and excellent momentum resolution. Using particle identification techniques that encompass $d E / d x$, time-of-flight [3, Multi-Cell Čerenkov 4] and a Ring Imaging Čerenkov (RICH)detector [5], MIPP is designed to identify charged particles at the $3 \sigma$ or better level in nearly all of its final state phase space. MIPP has acquired data of unparalleled quality and statistics for beam momenta ranging from $5 \mathrm{GeV} / \mathrm{c}$ to $90 \mathrm{GeV} / \mathrm{c}$ for 6 beam species $\left(\pi^{ \pm}, K^{ \pm}\right.$and $\left.p^{ \pm}\right)$on a variety of targets as shown in Figure 1.

An important aspect of MIPP data-taking was the measurement of particle production off the NuMI [6] target in order to minimize the sys-

\begin{tabular}{|c|c|c|c|c|c|c|c|c|c|c|c|c|}
\hline \multirow{2}{*}{\multicolumn{3}{|c|}{$\begin{array}{c}\text { Data Summary } \\
\text { 27 February } 2006 \\
\text { Target }\end{array}$}} & \multicolumn{10}{|c|}{$\begin{array}{c}\text { Acquired Data by Target and Beam Energy } \\
\text { Number of events, } x 10^{6}\end{array}$} \\
\hline & & & \multicolumn{9}{|c|}{$\mathbf{E}$} & \multirow[b]{2}{*}{ Total } \\
\hline $\mathrm{z}$ & Element & $\begin{array}{l}\text { Trigger } \\
\text { Mix }\end{array}$ & 5 & 20 & 35 & 40 & 55 & 60 & 65 & 85 & 120 & \\
\hline \multirow{3}{*}{0} & Empty & Normal & & 0.10 & 0.14 & & & 0.52 & & & 0.25 & 1.01 \\
\hline & $K$ Mass & No Int. & & & & 5.48 & 0.50 & 7.39 & 0.96 & & & 14.33 \\
\hline & Empty LH & Normal & & 0.30 & & & & 0.61 & & 0.31 & & \multirow{2}{*}{7.08} \\
\hline 1 & LH & Normal & 0.21 & 1.94 & & & & 1.98 & & 1.73 & & \\
\hline \multirow{2}{*}{4} & \multirow{2}{*}{$\mathrm{Be}$} & $p$ only & & & & & & & & & 1.08 & \multirow{2}{*}{1.75} \\
\hline & & Normal & & & 0.10 & & & 0.56 & & & & \\
\hline \multirow{3}{*}{6} & C & Mixed & & & & & & 0.21 & & & & \multirow{2}{*}{1.33} \\
\hline & C $2 \%$ & Mixed & & 0.39 & & & & 0.26 & & & 0.47 & \\
\hline & NuMI & $p$ only & & & & & & & & & 1.78 & 1.78 \\
\hline 13 & Al & Normal & & & 0.10 & & & & & & & 0.10 \\
\hline \multirow{2}{*}{83} & \multirow{2}{*}{ Bi } & $p$ only & & & & & & & & & 1.05 & \multirow{2}{*}{2.83} \\
\hline & & Normal & & & 0.52 & & & 1.26 & & & & \\
\hline 92 & $\mathbf{U}$ & Normal & & & & & & 1.18 & & & & 1.18 \\
\hline \multicolumn{3}{|c|}{ Total } & 0.21 & 2.73 & 0.86 & 5.48 & 0.50 & 13.97 & 0.96 & 2.04 & 4.63 & 31.38 \\
\hline
\end{tabular}

Figure 1. The data taken during the first MIPP run as a function of nucleus. The numbers are in millions of events. During the last month of the run, the Jolly Green Giant magnet coils developed shorts. This time was used to acquire data without the TPC for exploring the feasibility of measuring the charged kaon mass using the RICH radii.

tematics in the near/far detector ratio in the MINOS 6] experiment. MIPP also made measurements with proton beams off various nuclei for the needs of proton radiography [2].

Another physics motivation behind MIPP is to 
restart the study of non-perturbative QCD interactions. Currently available data are of poor quality, and sparsely populate the beam momentum, $p_{T}$, and atomic weight phase space that makes comparisons between different experiments difficult. The MIPP TPC [7] digitizes the charged tracks in three dimensions, obviating the need for track matching across stereo views. Coupled with the particle identification capability of MIPP, the data from MIPP would add significantly to our knowledge base of non-perturbative QCD. This would help test inclusive scaling relations and also scaling nuclear reactions.

\subsection{Experimental Setup}

We designed a secondary beam [8] specific to our needs. The resonantly extracted protons from the Fermilab Main Injector are transported down the Meson Center line. They impinge on a $20 \mathrm{~cm}$ long copper target producing secondary beam particles. This target is imaged onto an adjustable momentum selection collimator which controls the momentum spread of the beam. This collimator is re-imaged on to our interaction target placed next to the TPC. The beam is tracked using three beam chambers and identified using two differential Cerenkovs [9] filled with gas, the composition and the pressure of which can be varied within limits depending on the beam momentum and charge.

Figure 2 shows the layout of the apparatus. The TPC sits in a wide aperture magnet (the Jolly Green Giant) which has a peak field of 0.7 tesla. Downstream of the TPC are a 96 mirror multi-cell Čerenkov detector filled with $C_{4} F_{10}$ gas, and a time of flight system. This is followed by a large aperture magnet (Rosie) which runs in opposite polarity (at -0.6 tesla) to the Jolly Green Giant to bend the particles back into the Ring Imaging Čerenkov counter. The RICH has $\mathrm{CO}_{2}$ as the radiator and an array of phototubes of 32 rows and 89 columns [10. Downstream of the RICH we have an electromagnetic calorimeter [11] and a hadron calorimeter [12] to measure forward-going photons and neutrons. The electromagnetic calorimeter provides a means of distinguishing forward neutrons from photons and will also serve as a device to measure the electron content of our beam at lower energies, which will be useful for measuring cross sections.

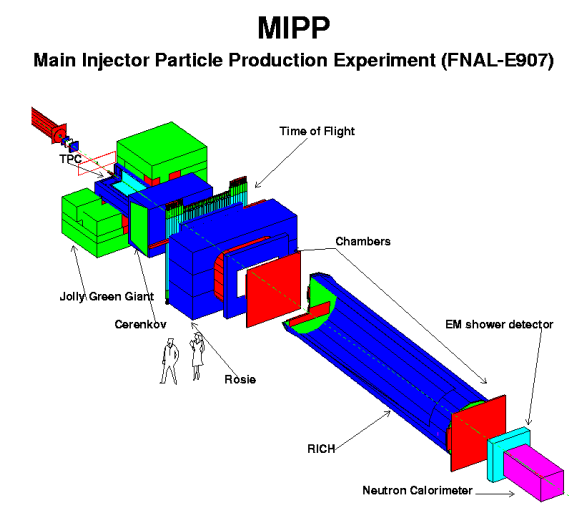

Figure 2. The experimental setup. The picture is a rendition in Geant3, which is used to simulate the detector.

MIPP uses $d E / d x$ in the TPC to separate pions, kaons and protons for momenta less than $\approx$ $1 \mathrm{GeV} / \mathrm{c}$ and the time of flight array of counters to do the particle identification for momenta less than $2 \mathrm{GeV} / \mathrm{c}$. The multi-cell Cerenkov detector [4] contributes to particle identification in the momentum range $\approx 2.5 \mathrm{GeV} / \mathrm{c}-14 \mathrm{GeV} / \mathrm{c}$ and the RICH [5] for momenta higher than this. By combining information from all counters, we get the expected particle identification separation for $K / p$ and $\pi / K$ as shown in Figure 3. It can be seen that excellent separation at the $3 \sigma$ or higher level exists for both $K / p$ and $\pi / p$ over almost all of phase space. Tracking of the beam particles and secondary beam particles is accomplished by a set of drift chambers [13] and proportional chambers [14] each of which have 4 stereo layers.

\subsection{Some results from Acquired data}

Figure 4 shows the pictures of reconstructed tracks in the TPC obtained during the data- 

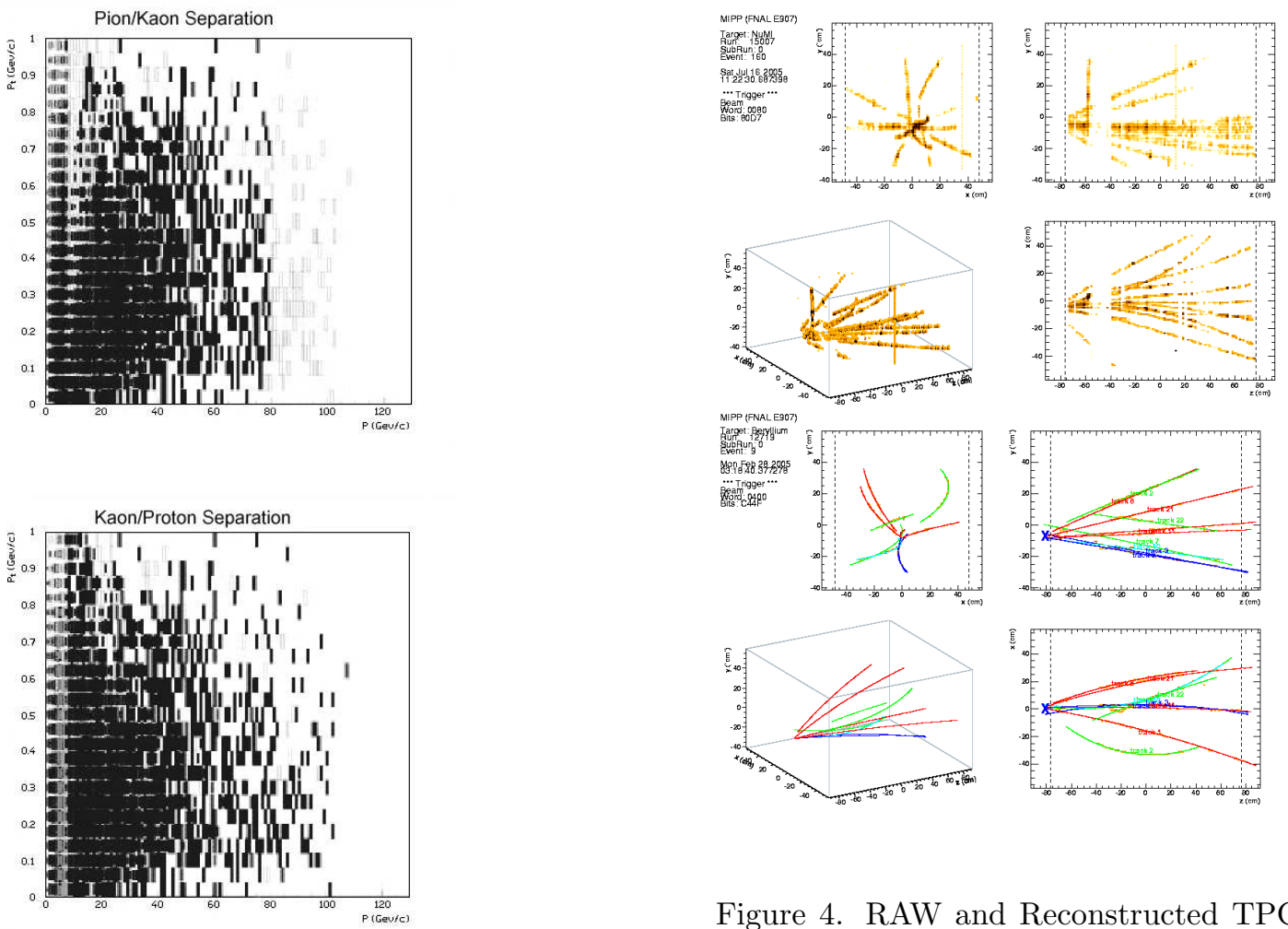

taking run. The tracks are digitized and fitted as helices in three dimensions. Extrapolating three dimensional tracks to the other chambers makes the pattern recognition particularly easy.
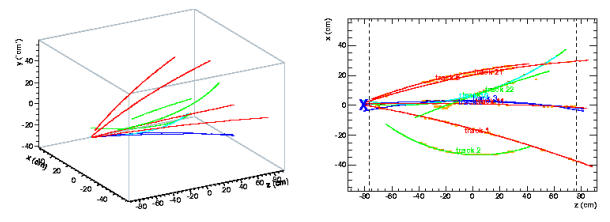

Figure 4. RAW and Reconstructed TPC tracks from two different events.

Figure 3. Particle identification plots for pion/kaon separation and for kaon/proton separation as a function of the longitudinal and transverse momentum of the outgoing final state particle. Black indicates separation at the $3 \sigma$ level or better and grey indicates separation at the $1-3 \sigma$ level. The boxes at largest values of the longitudinal momenta suffer from lack of kaon statistics.

Figure 5 shows the distribution of $d E / d x$ of tracks measured in the TPC as a function of the track momentum in a preliminary analysis of $\mathrm{p}$ Carbon data. The TPC is capable of separating pions, protons and kaons in the momentum range below $\approx 1 \mathrm{GeV} / \mathrm{c}$. Figure 6 shows events with multiple rings in the RICH counter. Figure 7 shows the histogram of ring radii for a $+40 \mathrm{GeV}$ secondary beam. There is clean separation between pions, kaons and protons and their relative abundances [15] match expectations. Applying the particle identification trigger from the beam Cerenkovs enables us to separate the three particle species cleanly. The kaons which form 


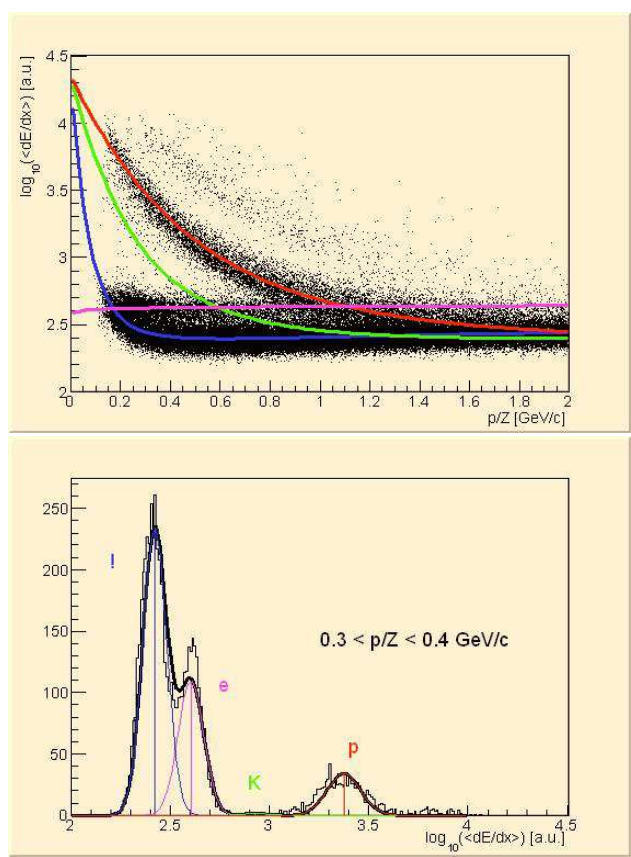

Figure 5. Preliminary $\mathrm{dE} / \mathrm{dx}$ distributions in the TPC The scatter plot shows the electron, pion, kaon and proton peaks in the distribution as a function of the lab momentum for p-Carbon data. The second plot is the projection on the $\mathrm{dE} / \mathrm{dx}$ axis for a momentum slice $0.3 \mathrm{GeV} / \mathrm{c}$ to $0.4 \mathrm{GeV} / \mathrm{c}$.

$4 \%$ of the beam are cleanly picked out by the beam Čerenkov with very simple selection criteria. These can be made much more stringent with offline cuts to produce a very clean kaon beam.

The ring radius of the particle contains information on the mass of the particle. The pion and proton masses are very well known. The charged kaon mass, however, currently has measurement uncertainties of the order of $60 \mathrm{keV}$. Improving the precision of both charged kaon masses will pay dividends in rare $\mathrm{K}$ decay experiments involving charged kaons where the matrix elements depend on the kaon mass raised to large powers. Towards the end of our physics run, when the Jolly Green Giant magnet coils failed, we switched off the TPC and acquired data at the
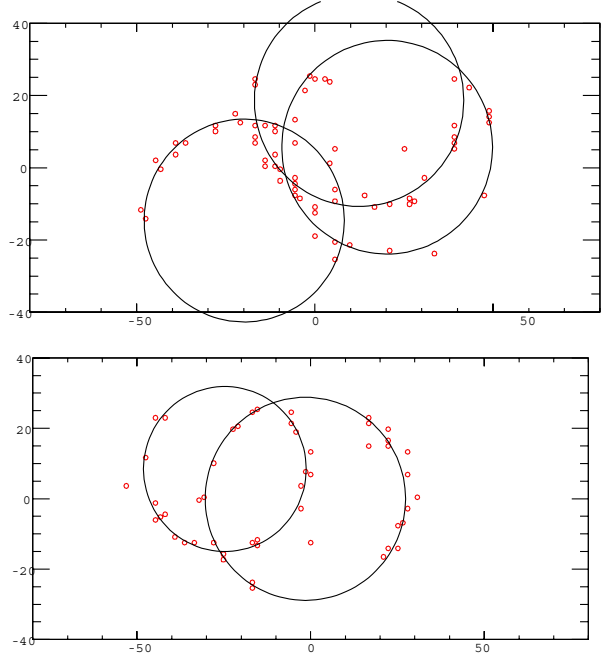

Figure 6. Examples of events with rings in the $\mathrm{RICH}$ counter for a $40 \mathrm{GeV} / \mathrm{c}$ beam. The $\mathrm{x}$ and $\mathrm{y}$ axes are in $\mathrm{cm}$.

rate of $300 \mathrm{~Hz}$ to investigate how well we can measure the charged kaon mass. These events, whose statistics are indicated in Figure 1, are currently being analyzed to evaluate the systematics involved in such a measurement.

\subsubsection{NuMI target measurements}

MIPP took 1.75 million events using $120 \mathrm{GeV} / \mathrm{c}$ primary beam protons impinging on the NuMI (spare) target. These events will play a crucial role in the prediction of neutrino fluxes in the NuMI beamline and will enable the MINOS experiment to control the systematics in the near/far detector ratios as well as helping them understand the near detector performance. Figure 8 shows a radiograph of the MIPP measurements of the MINOS target. The graphite slabs and cooling tubes can be seen. These events were obtained during the commissioning phase of this target measurement where the beam was not yet fully focused and aligned on the target. The 1.75 Million events on the NuMI target were obtained after the beam was aligned and centered on the target. Figure 9 shows the rich ring radii 

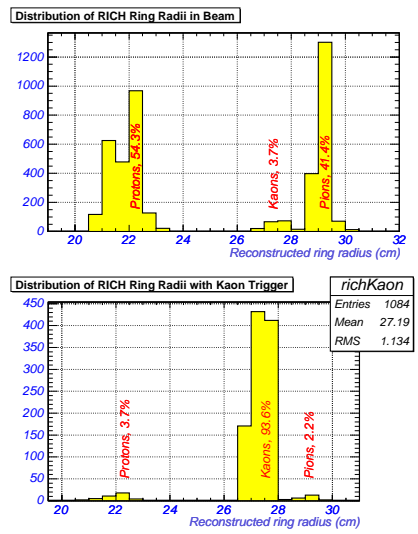
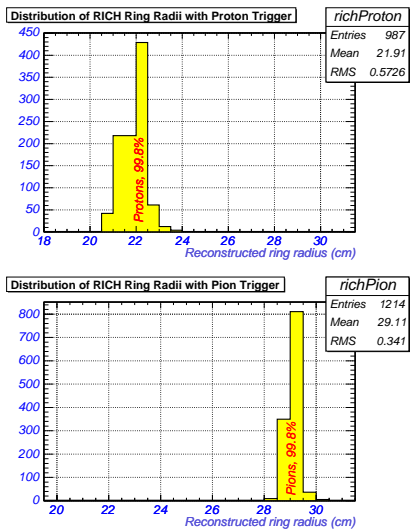

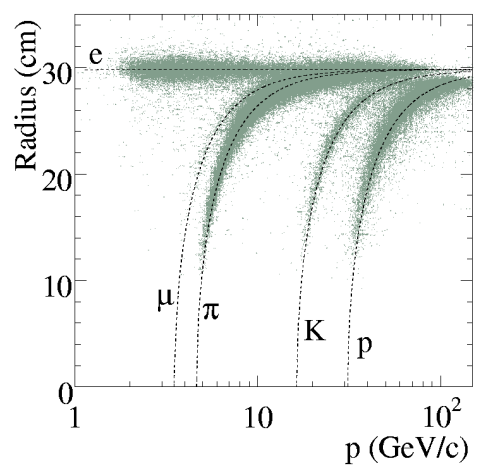

Figure 7. An example of a $40 \mathrm{GeV} / \mathrm{c}$ primary beam (non-interacting) trigger. The RICH identifies protons, kaons and pions by the ring radii. The beam Cerenkov detectors can be used to do the same. When the beam Cerenkov identification is used, one gets a very clean separation of pions, kaons and protons in the RICH.

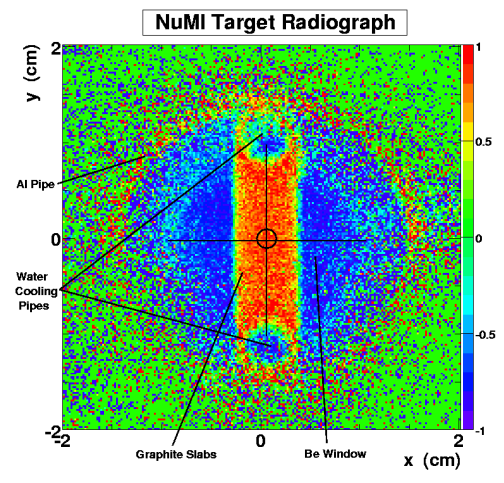

Figure 8. Radiograph of the MINOS target. The beam direction is perpendicular to the paper.

vs momentum of positive tracks originating from the NuMI target. Superimposed are the curves for known particles. This shows the excellent particle identification of the MIPP detector for forward going particles.

\subsection{Particle production on Nitrogen and the question of Cosmic Ray Showers in the Atmosphere}

With the upgraded MIPP, we propose to measure particle production on a cryogenic nitrogen target using positive and negative beams, which is needed by experiments measuring cosmic ray air showers (Pierre Auger, HiRes etc) and also atmospheric neutrinos (Amanda, Ice Cube, HyperK etc). The problem is illustrated in a recent paper [16] which simulates the air showers produced by protons of $10^{6} \mathrm{GeV}$ energy in the atmosphere. The shower goes through several generations of interactions and produce pions and kaons that decay to produce muons and neutrinos. The muons and neutrinos are observed in the detectors and are termed the daughter particles. The mesons that produced the muons and neutrinos are termed the mother particles and the particles that interacted in the atmosphere to produce the mother particles are termed the grandmother particles in the jargon.

Figure 10 shows the energy spectrum of the grandmother particles $(\pi, K$ and $p$ in an air shower that are produced by a primary proton of $10^{6} \mathrm{GeV}$. The spectrum for the pions peaks at $100 \mathrm{GeV}$ and the kaons and protons at somewhat 
higher energies. These particles interact with the nitrogen (and oxygen) in the atmosphere to produce the atmospheric neutrinos and muons. In other words, the beam energies available at MIPP are relevant to the simulation of the cosmic ray air showers. The muon flux measurement is a critical component of estimating the energy scale of the cosmic ray shower. MIPP measurements thus will help reduce the systematics in the cosmic ray energy scale measurements. As the primary cosmic ray energy increases, the peaks in this plot do not shift to higher energies. Understanding the shower systematics at the peak of this spectrum (i.e MIPP energies) will help the energy systematics of cosmic rays of all energies. Figure 11

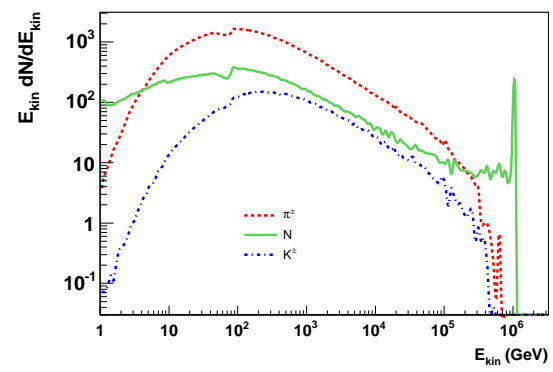

Figure 10. The energy distribution of the grandmother particles in a vertical air shower produced by $10^{6} \mathrm{GeV}$ proton interacting in the atmosphere as a function of particle type. These particles interact further in the atmosphere to produce more particles which then decay into muons and neutrinos. The muons are detected at a distance of 0-500 meters from the shower center at ground level. It can be seen that these spectra peak at energies relevant to the MIPP energy scale.

shows the distribution of grandmother particles at different lateral distances from the shower center for all particle types. For the lower energy interactions, the simulation code Gheisha is used to simulate the interactions of the particles with the atmosphere. For higher energy interactions, the simulation code QGSJET 01 is used. The sharp break in the spectra at $100 \mathrm{GeV}$ is where the two codes meet and disagree at places by a factor of two. This illustrates the problem. These codes at present are "tuned" on single arm spectrometer data and disagree with each other.

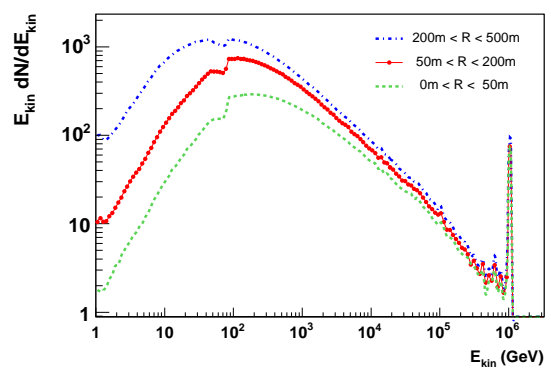

Figure 11. The energy distribution of the grandmother particles in a vertical air shower produced by $10^{6} \mathrm{GeV}$ proton interacting in the atmosphere as a function of distance from the shower center. The spectrum peaks at energies relevant to the MIPP energy scale.

Most of the data acquired to date of relevance to air showers are over 25 years old and were obtained using Beryllium targets and single arm spectrometers resulting in a discrete angular coverage. MIPP will measure the outgoing pion and kaon spectrum for proton and pion beams in its full secondary beam momentum range. These cross sections are the most important in simulating cosmic ray showers in the atmosphere. In addition, it will also have kaon and antiproton interactions on nitrogen of which virtually nothing is known. Being an open geometry experiment, the MIPP angular coverage will be continuous, not discrete.

The need for MIPP data is recognized by the cosmic ray community, some of whom have joined the experiment. 


\subsubsection{MIPP Measurement of $\pi / K$ ratios}

Because of its excellent particle identification capabilities, MIPP upgrade will measure the ratio of charged kaons to pions as a function of $p_{L}, p_{T}$ of the final state particle. This measurement is of importance to both the NuMI target measurements and the atmospheric neutrino measurements, since the charged K's produce $\nu_{e}^{\prime} s$ which are a background to the oscillation search $\nu_{\mu} \rightarrow \nu_{e}$.

\section{MIPP Upgrade Proposal}

In October 2006, MIPP proposed to upgrade its detector by increasing the data acquisition speed of its TPC from $20 \mathrm{~Hz}$ to $3000 \mathrm{~Hz}$ using the ALTRO/PASA chips developed for the ALICE collaboration [17]. With this large factor in data acquisition speed, it becomes possible to acquire 5 million events in a single day of running. The electronics of the rest of the detector will also be upgraded to run at this speed. We propose to use a hemisphere of the plastic ball detector to measure the target recoil particles. This enables us to measure particle production on 30 of the most common nuclei found in particle physics detectors and improve the hadronic shower simulator programs. It will also permit us to study nonperturbative QCD in unprecedented detail. The baryon resonance spectrum can be investigated up to $3 \mathrm{GeV} / \mathrm{c}^{2}$, using pion and kaon beams in the momentum range $1 \mathrm{GeV} / \mathrm{c}-5 \mathrm{GeV} / \mathrm{c}$. The decision on the upgrade proposal has been deferred till analysis results from the present run are available and the collaboration has been strengthened further.

\subsection{Upgrade Run Plan}

We propose to conduct the upgrade running in three phases. In the first phase, we will acquire data on the neutrino targets, liquid nitrogen and take particle production measurements on 12 thin nuclear targets ( 5 million events per nucleus) which will improve the quality of hadronic shower simulation programs. The number of events acquired in each target is indicated in table 1.

During phase 2, we plan to complete the remaining 18 nuclei of the A-List, as detailed in the

\begin{tabular}{ccc}
\hline Target & $\begin{array}{c}\text { Events } \\
\text { (Millions) }\end{array}$ & $\begin{array}{c}\text { Running Time } \\
\text { (Days) }\end{array}$ \\
\hline NuMI target 1 & 10 & 2 \\
NuMI target 2 & 10 & 2 \\
Liquid Hydrogen & 20 & 4 \\
Liquid Nitrogen & 10 & 2 \\
12 Nuclei & & \\
$D_{2}, \mathrm{Be}, \mathrm{C}, \mathrm{Al}, \mathrm{Si}, \mathrm{Hg}$, & & \\
Fe,Ni,Cu,Zn,W,Pb & 60 & 12 \\
Total Events & 110 & 22 \\
\hline
\end{tabular}

Table 1

Phase 1 Run Plan.

\begin{tabular}{ccc}
\hline Target & $\begin{array}{c}\text { Events } \\
\text { (Millions) }\end{array}$ & $\begin{array}{c}\text { Running Time } \\
\text { (Days) }\end{array}$ \\
\hline 18 Nuclei & & \\
Li,B, $O_{2}, \mathrm{Mg}, \mathrm{P}$, & & \\
S,Ar,K,Ca,Ni,Nb,Ag, & & 18 \\
Sn,Pt,Au,Pb,Bi,U & 90 & \\
10 Nuclei B-list & & \\
Na,Ti,V, Cr,Mn,Mo, & & 10 \\
I, Cd, Cs, Ba & 50 & 28 \\
Total Events & 140 & \\
\hline Table 2 & &
\end{tabular}

Phase 2 Run Plan.

section on hadronic shower simulation and then proceed with the B-list if there is need. The second phase of running is detailed in table 2

During phase 3, we plan to go into the tagged neutral beam mode, where we run the liquid hydrogen target and allow the ILC calorimetry to run simultaneously in place of the MIPP calorimeter to study the response of the ILC calorimeters to tagged neutral beams.

\section{Conclusions}

MIPP has acquired high quality particle production data which it is busy analyzing. The MIPP upgrade will improve quality and statistics data by an order of magnitude by speeding up the upgrade. Collaborators are welcome. 


\section{REFERENCES}

1. The main MIPP web page is at http://ppd.fnal.gov/experiments/e907/. Information on the MIPP collaboration may be found using links there.

2. The MIPP proposal and Addendum to the proposal may be found at http://ppd.fnal.gov/experiments/e907 /Proposal/E907_Proposal.html

3. The Time of Flight detector was fabricated by MIPP and consists of an array of $10 \mathrm{~cm} \times 10 \mathrm{~cm}$ scintillators and $5 \mathrm{~cm} \times 5 \mathrm{~cm}$ scintillators. See http://ppd.fnal.gov/experiments/e907 /TOF/TOF.html for a detailed description of the detector.

4. The multi-cell Cerenkov detector was first used in BNL experiment E766, then in Fermilab experiment E690 followed by other BNL experiments.

5. The details of the SELEX RICH construction and performance may be found at J. Engelfried et al., Nucl. Instr. and Meth. A43,53(1999). We have replaced the front end electronics, and done extensive work on the safety systems. MIPP uses $\mathrm{CO}_{2}$ gas as the radiator for the $\mathrm{RICH}$.

6. NuMI stands for Neutrinos at the Main Injector and refers to the Fermilab Main Injector neutrino beam. MINOS is the first experiment to utilize this beam MINOS proposal may be found at "P-875: A long baseline neutrino oscillation experiment at Fermilab", E. Ables et al.; FERMILABPROPOSAL-P875,(1995). See also their website at http://www-numi.fnal.gov/

7. The TPC was built by the BEVALAC group at Berkeley in the 1990's and used effectively at several Brookhaven experiments (e.g. E910) and then donated to Fermilab by LBNL for use in MIPP. See, G. Rai et al., IEEE Trans.Nucl.Sci.37,56(1990); LBL28141.

8. "Beamline design for particle production Experiment, E907 at FNAL", C. Johnstone et al., Proceedings of the PAC03 conference. So successful has the performance of this beam- line been that the design has been adopted for use in the M-Test beamline upgrade for providing testbeams at Fermilab.

9. For more details on the beam Čerenkov system, see http://ppd.fnal.gov/experiments/e907/Beam /BeamCerenkov/BeamCerenkov.html

10. In 2004 , we lost $20 \%$ of the phototubes in the RICH due to a fire in one of the phototube bases. This does not impact adversely on our pattern recognition, since the Čerenkov angle is large and there is plenty of light over most of our momentum range.

11. The EM calorimeter was fabricated by MIPP and uses lead as the radiator and an array of proportional tubes with $2.54 \mathrm{~cm}$ wire spacing as the readout. It has 10 radiation lengths and has 10 longitudinal segments.

12. Theiron/scintillator fiber hadron calorimeter is recycled from the HyperCP collaboration. It has 9.7 interaction lengths and has 4 longitudinal and 2 transverse segmentations.

13. We have reused beam and drift chambers from the E690 collaboration. D.C.Christian et al., Nucl. Instr. and Meth. A345,62 (1994).

14. The large proportional chambers straddling the RICH find their use again after having been used by numerous previous experiments. M. De Palma et al., Nucl. Instr. and Meth. 216 (1983) 393-397.

15. A. Malensek, Fermilab Technical Memo FN341, 1981. (unpublished).

16. "Muon production in extensive air showers and its relation to hadronic interactions", C. Meurer et. al., to appear in the proceedings of the International Conference on Interconnection between High Energy Physics and Astroparticle Physics: From Colliders to Cosmics Rays, Prague, Czech Republic, 7-13 Sep 2005. astro-ph/0512536.

17. The details of the MIPP upgrade Proposal (P-960) can be found at http://ppd.fnal.gov/experiments/e907/notes/ MIPPnotes/public/pdf/MIPP0138/MIPP0138.pdf 